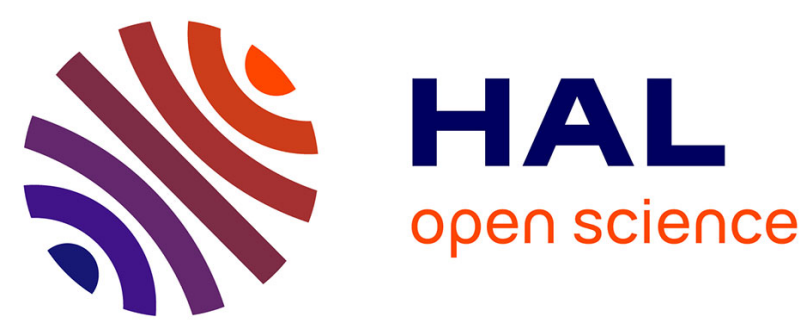

\title{
Optimal integrated maintenance/production policy for randomly failing systems with variable failure rate
}

\author{
Hajej Zied, Sofiene Dellagi, Nidhal Rezg
}

\section{To cite this version:}

Hajej Zied, Sofiene Dellagi, Nidhal Rezg. Optimal integrated maintenance/production policy for randomly failing systems with variable failure rate. International Journal of Production Research, 2011, pp.1. 10.1080/00207543.2010.528063 . hal-00660538

\section{HAL Id: hal-00660538 \\ https://hal.science/hal-00660538}

Submitted on 17 Jan 2012

HAL is a multi-disciplinary open access archive for the deposit and dissemination of scientific research documents, whether they are published or not. The documents may come from teaching and research institutions in France or abroad, or from public or private research centers.
L'archive ouverte pluridisciplinaire HAL, est destinée au dépôt et à la diffusion de documents scientifiques de niveau recherche, publiés ou non, émanant des établissements d'enseignement et de recherche français ou étrangers, des laboratoires publics ou privés. 


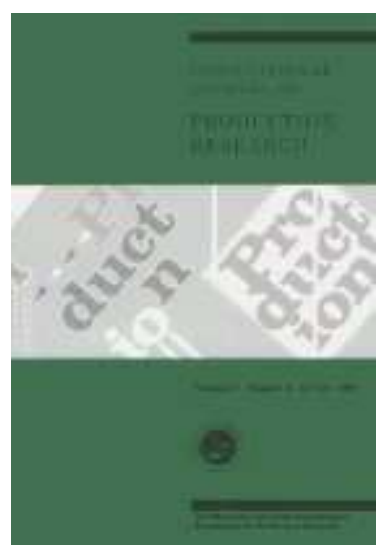

\section{Optimal integrated maintenance/production policy for randomly failing systems with variable failure rate}

\begin{tabular}{|c|c|}
\hline Journal: & International Journal of Production Research \\
\hline Manuscript ID: & TPRS-2010-IJPR-0856 \\
\hline Manuscript Type: & Original Manuscript \\
\hline $\begin{array}{r}\text { Date Submitted by the } \\
\text { Author: }\end{array}$ & 27-Aug-2010 \\
\hline Complete List of Authors: & $\begin{array}{l}\text { Zied, Hajej; LGIPM } \\
\text { DELLAGI, Sofiene; Université Paul Verlaine, Metz } \\
\text { REZG, Nidhal; Universite Paul Verlaine, Metz }\end{array}$ \\
\hline Keywords: & $\begin{array}{l}\text { PREVENTIVE MAINTENANCE, MAINTENANCE PLANNING, } \\
\text { PRODUCTION PLANNING, MANUFACTURING STRATEGY, } \\
\text { OPTIMIZATION }\end{array}$ \\
\hline Keywords (user): & PREVENTIVE MAINTENANCE, PRODUCTION PLANNING \\
\hline
\end{tabular}

\section{SCHOLARONE"}

Manuscripts 


\title{
Optimal integrated maintenance/production policy for randomly failing systems with variable failure rate
}

\author{
Hajej Zied, Dellagi Sofiene, Rezg Nidhal \\ LGIPM-INRIA, Université Paul Verlaine, Metz, France \\ \{hajej, dellagi,rezg\}@univ-metz.fr
}

\begin{abstract}
This paper deals with combined production and maintenance plans for a manufacturing system satisfying a random demand. We first establish an optimal production plan which minimizes the average total inventory and production cost. Secondly, using this optimal production plan, and taking into account the deterioration of the machine according to its production rate, we derive an optimal maintenance schedule which minimizes the maintenance cost. A numerical example illustrates the proposed approach, this analytical approach, based on a stochastic optimization model and using the operational age concept, reveals the significant influence of the production rate on the deterioration of the manufacturing system and consequently on the integrated production/maintenance policy.
\end{abstract}

Keywords

Failure rate, Maintenance strategies, Operational age, Linear quadratic model, Maintenance scheduling, Production plan.

\section{Introduction}

Recently, maintenance and production scheduling using stochastic optimal control techniques has drawn much attention among researchers. Due to the complexity of the manufacturing systems, decisions pertaining to marketing, production and maintenance have traditionally been treated separately. Clearly, however, analyzing these decisions simultaneously is more realistic and useful from a practical point of view. Accordingly, this study seeks to find the joint optimal production and maintenance strategy for a randomly failing manufacturing system which must satisfy a random product demand over future periods. This is indeed a complex task due to the various uncertainties caused by exogenous and endogenous factors. While exogenous factors are typically due to demand randomness, an example of an endogenous factor would be the availability of the production system. As a direct effect of these random elements, the inventory variable cannot be computed precisely, giving rise to the need to adopt a stochastic optimal control approach. Moreover, it is interesting to develop an intelligent optimal maintenance strategy considering the deterioration of the manufacturing system as a function of the production rate. Little research has been conducted in this area. Akella and Kumar (1986) formulated a one-machine one-part-type production problem as a stochastic optimal control problem, in which the part demand is assumed to be constant, the state of the machine is assumed to be a two-state continuoustime Markov chain, and the objective function is a discounted inventory/shortage cost over an infinite time horizon. 
(Silva and Wagner, 2004) deal with a chance-constrained stochastic production-planning problem under hypotheses of imperfect information of inventory variables. The optimal production plan is obtained by the minimizing of the expected cost. Barták et al. (2009) describe a constraint programming approach solving scheduling problems with earliness and tardiness costs. In the same vein, Kelle et al. (1994) considered a single-product with random demand along with a single-machine with setups in the process industry. They formulated a model that incorporates mean and standard deviation of demand in each period. Though only one product was being made, start-ups after periods of idleness required significant setups.

In the situation of interest here, the stochastic nature of the system is due to machines which are subject to breakdowns and repairs or maintenance actions. The traditional maintenance strategies proposed in the literature are mainly policies involving the critical age of a machine or a set of machines. These policies are based on models describing the equipment failure law. The basic assumptions related to repair efficiency are known as minimal repair or as bad as old (ABAO) and perfect repair or as good as new (AGAN). In the ABAO case, each repair restores the system to the operating state to leaves it with the same failure rate level, he had before failure. In the AGAN case, each repair is perfect and restores the system was new. Obviously, reality lies somewhere between these two extreme cases: standard maintenance reduces the failure rate but does not return the system to the as good as new condition. This is sometimes known as imperfect or better-thanminimal repair. Along these lines, Brown and Proschan (1983) considered a model in which a perfect repair occurs with probability $p$ whereas a minimal repair occurs with probability (1-p). Another class of models of interest is the one of virtual age models proposed by Kijima (1988). Usually, these models are defined by the conditional distributions of successive inter-failure times.

The cost/time of maintenance/repair is supposed to be known and consequently the impact of a maintenance/failure can be analyzed. Under these conditions, it can be shown that the optimal policy is of the critical age type which consists in carrying out a preventive maintenance action at its critical age. In this context, Boukas and Yang (1996) assumed the simultaneous planning of production and maintenance in a flexible manufacturing system. The system is composed of a single machine subject to random failures which produces a given commodity. The probability of machine failure is supposed to be an increasing function of its age. The objective is to minimize the discounted inventory and maintenance cost subject to meeting the demand.

Moreover, under production control policies such as just-in-time, which requires the availability of machines at the right time, an integrated approach of maintenance and production control becomes essential. In this context, Rezg et. al. (2004) proposed a method for the joint optimization of preventive maintenance and stock control in a production line made up of $N$ machines. Rezg et al. (2008) similarly presented a mathematical model and a numerical procedure for determining simultaneously an optimal inventory control policy and an age-based preventive maintenance policy for a randomly failing production system. Boukas and Haurie (1990) considered a system which has two machines with age-dependent failure rates and where a preventive maintenance decision must be made. They used a numerical method to evaluate the optimal control policy and showed that in their context the optimal hedging surfaces can be defined to represent the optimal production policies. Van der Dyun Schouten and Vanneste (1995) proposed an age-based preventive maintenance policy considering the capacity of a buffer stock between two machines. Moreover, maintenance/production strategies taking into account the context of a subcontractor are studied by Dellagi, et al. (2007), while Cheung 
Fig 1. Problem description

During the horizon $H$, machine $M$ is subject to random failure. The probability density function of time to failure is $f(t)$, while the failure rate $\lambda(t)$ is increasing in both time and production rate $u(t)$. Failures of machine $M$ can be reduced through preventive maintenance activities. Preventive maintenance (PM), usually scheduled periodically at certain time intervals, is a policy aimed at improving the overall reliability and availability of a system. Ideally, one would like to define a PM policy such that the overall cost of system failure, maintenance, and replacement during its production horizon $H$ is minimized.

Under the constraint that the total time needed to perform both maintenance activities (preventive and corrective) is not greater than the finite horizon $H$, the Cox model (Cox, 1972), given below for a period $k$ during an horizon 
$H$, provides an estimate of the treatment effect on survival after adjustment for other explanatory variables. Thus he establishes a parametric relationship between risk factors (related to the operational and environmental conditions of each period) and the hazard rate. The model relies mainly on the assumption of proportional hazards, which implies that each factor affects the life steadily over time.

Let

$\lambda_{0}(t)$ the hazard rate for nominal conditions

$g\left(u_{k}\right)$ the risk function of period $k$

$\lambda\left(t, u_{k}\right)$ the hazard rate representing the instantaneous failure risk at time $t$ under condition $u_{k}$

For a period $k$, the Cox model is given by:

$$
\lambda\left(t, u_{k}\right)=\lambda_{0}(t) \cdot g\left(u_{k}\right)
$$

Our first objective is to establish an economical production plan satisfying the random demand. Secondly, using this optimal production plan, we establish the optimal preventive maintenance plan. The use of the optimal production plan as an input to the maintenance study is justified by the influence which the production rate at each period exerts on the failure rate of the machine. Since the Cox model is used to define the failure law, each period has its distinct failure rate. Meantime, the operational and environmental conditions will impact the optimal scheduling of maintenance actions through the minimization of the average number of failures. The cost and duration of a PM activity are respectively assumed to be strictly lower than the cost and duration of a corrective maintenance action.

\section{II.1. Notation}

The main decision variables, cost coefficients and parameters associated with the stochastic problem at hand are listed below:

$H$ : finite production horizon

$\Delta \mathrm{t}$ : period length of production

$s(k)$ : inventory level at the end of the period $k(k=1, \ldots \ldots ., H / \Delta t)$

$u(k)$ : production level at period $k(k=1, \ldots \ldots, H / \Delta t)$

$d(k)$ : demand level at period $k(k=1, \ldots \ldots ., H / \Delta t)$

$C_{p r}:$ unit production cost

$C_{s}$ : holding cost of a product unit during the period $k$

$f(t)$ : probability density function of time to failure for the machine

$R(t)$ : reliability function

$C p$ : preventive maintenance action cost

$C_{c}$ : corrective maintenance action cost

$m u$ : monetary unit

$U_{\max }$ : maximal production rate

$Z$ : total expected cost of production and inventory over the finite horizon $H$

$C$ : total expected maintenance cost per time unit 
$\alpha$ : probabilistic index (related to customer satisfaction)

\section{II.2. Problem formulation}

It is assumed that the horizon $H$ is partitioned equally into $N$ periods of length $H / \Delta t$. Let $\left\{f_{k}, k=1, \ldots ., N\right\}$ represent holding and production costs (they will be formulated in the next subsection), and $E\{\}$ denotes the mathematical expectation operator. The following aggregate sequential stochastic linear programming problem provides an optimal production plan over the planning horizon:

$$
\min _{u(k)} E\left\{\sum_{k=0}^{N-1} f_{k}(s(k), u(k))+f_{N}(s(N))\right\}
$$

Subject to:

$$
\begin{gathered}
s(k+1)=s(k)+u(k)-d(k) \quad k=0,1, \ldots, N-1 \\
\text { Prob }[s(k+1) \geq 0] \geq \alpha \quad k=0,1, \ldots, N-1 \quad(2) \\
0 \leq u(k) \leq U_{\text {max }} \quad k=0,1, \ldots, N-1 \quad
\end{gathered}
$$

Constraint (1) defines the inventory balance equation for each time period. The constraint (2) imposes the service level requirement for each period as well as a lower bound on inventory variables so as to prevent stockouts. Note that the non-negative lower limit in (2) represents a safety stock. Finally, the last constraint defines an upper bound on the production level during each period $k$.

\section{II.3. The stochastic production policy}

The purpose of this subsection is to develop and optimize the expected production and holding costs $E\{f()$.$\} over$ the finite time horizon $H$. As mentioned above, the demand $d$ is a random variable with mean $\hat{d}(k)$ and standard-deviation $\sigma_{d}(k)$, which are known for each period $k$. The randomness of demand turns the inventory balance equation (1) into a stochastic process that also has a probability distribution. Since demand must be satisfied at the end of each period, the problem can be formulated as a linear-stochastic optimal control problem under a threshold inventory level constraint, as follows:

$$
u^{*}=\min _{u}(Z(u))
$$

with:

$$
u=(u(1), u(2), \ldots u(k) \ldots u(N))
$$

The model is described by a hybrid state with continuous component, namely the inventory level as given by equation (1) above, with $s(0)=s_{0}$, where $s_{0}$ is the given initial inventory. 
The expected production and holding costs for period $k$ are given by:

$$
f_{k}(s(k), u(k))=C_{s} \cdot E\left\{\left(s(k)^{2}\right)\right\}+C_{p r} \cdot\left(u(k)^{2}\right)
$$

Remark:

The use of quadratic costs allows penalizing both excess and shortage of inventory.

The total expected cost of production and inventory over the finite horizon $H$ can then be expressed as:

$$
F(u)=\sum_{k=0}^{N} f_{k}(u(k), s(k))=C_{s} \times E\left\{\left(s(N)^{2}\right)\right\}+\sum_{k=0}^{N-1}\left[C_{s} \times E\left\{s(k)^{2}\right\}+C_{p r} \times u(k)^{2}\right]
$$

Remark:

$(u(N))^{2}$ is not included in the cost formulation because we don't consider the production order at the end of the horizon $H$.

Thus the problem becomes:

$$
\text { (P1) : } \min _{u}\left[C_{s} \times E\left\{\left(s(N)^{2}\right)\right\}+\sum_{k=0}^{N-1}\left(C_{s} \times E\left\{s(k)^{2}\right\}+C_{p r} \times u(k)^{2}\right)\right]
$$

Subject to:

$$
\begin{gathered}
s(k+1)=s(k)+u(k)-d(k) \quad k=0,1, \ldots, N-1 \\
\operatorname{Prob}[s(k+1) \geq 0] \geq \alpha \quad k=0,1, \ldots, N-1 \\
0 \leq u(k) \leq U_{\max } \quad k=0,1, \ldots, N-1
\end{gathered}
$$

The following figure describes dynamic system evolution in discrete time:

Fig 2. Discrete time

\section{II.4. Maintenance policy}

The maintenance strategy under consideration is the well known preventive maintenance policy with minimal repair at failure (Faulkner, 2005). Perfect preventive maintenance is performed periodically at times $k . T$, $k=0,1, \ldots, N$, following which the unit is as good as new. Whenever a failure occurs between preventive maintenance actions, the system undergoes a minimal repair to allow it to continue operating during the current period and hence the failure rate is undisturbed. It is assumed that the repair and replacement times are negligible. It has been proved in the literature that the average maintenance total cost per time unit is expressed as follows:

$$
C_{T}=\frac{C_{p}+C_{c} \times \int_{0}^{T} \lambda(t) d t}{T}
$$




\section{Analytical determination of the joint production-maintenance policy}

\section{III.1.Production Policy}

This section focuses on determining the optimal production plan characterized by the best combination of production rates and inventory levels so as to minimize the total costs over the planning horizon $H$. In practice, the model provides a linear decision rule for inventory and production bearing in mind the requirement of satisfying the random demand.

Recall that our problem formulated in subsection II.3 is:

$$
\min _{u} F(u)=\min _{u}\left[C_{s} \times E\left\{\left(s(N)^{2}\right)\right\}+\sum_{k=0}^{N-1}\left(C_{s} \times E\left\{s(k)^{2}\right\}+C_{p r} \times u(k)^{2}\right)\right]
$$


Subject to:

$$
\begin{gathered}
s(k+1)=s(k)+u(k)-d(k) \quad k=0,1, \ldots, N-1 \\
\operatorname{Prob}[s(k+1) \geq 0] \geq \alpha \quad k=0,1, \ldots, N-1 \\
0 \leq u(k) \leq U_{\text {max }} \quad k=0,1, \ldots, N-1
\end{gathered}
$$

Solving such a sequential stochastic linear programming problem under constraints is generally difficult. Let us proceed by transforming the stochastic problem into an equivalent deterministic problem which will then be easier to solve.

$\underline{\text { Transformation to an equivalent deterministic problem }}$

- The objective function :

We can simplify the expected value of the production/inventory costs of eq. (8) as follows:

\section{Lemma1:}

$$
F(u)=C_{s} \times\left(\hat{s}(N)^{2}\right)+\sum_{k=0}^{N-1}\left[C_{s} \cdot \hat{s}(k)^{2} \times+C_{p r} \times u(k)^{2}\right]+C_{s} \times\left(\sigma_{d}\right)^{2} \times \frac{N(N+1)}{2}
$$

Where $\hat{S}(k)$ represents mean stock level at the end of period $k$

- The inventory balance equation:

Letting $d_{k}=\hat{d}_{k}$, the inventory balance equation (1) can be converted to:

$$
\hat{s}(k+1)=\hat{s}(k)+u(k)-\hat{d}(k)
$$

Since $u(k)$ is constant for each interval $\Delta t$, we have $\hat{u}(k)=u(k)$ and $\operatorname{Var}(u(k)=0)$

Proof of equation (9):

The inventory variable $s(k)$ is statistically described by its mean $E\{s(k)\}=\hat{s}(k)$ and variance $\operatorname{Var}(s(k))$

$$
E\left\{(s(k)-\hat{s}(k))^{2}\right\}=\operatorname{Var}(s(k))
$$

The balance equation (1) can be converted into an equivalent inventory balance equation, as follows

$$
\begin{aligned}
(1) & \Rightarrow E\{s(k+1)\}=E\{s(k)+u(k)-d(k)\} \\
& \Rightarrow \hat{s}(k+1)=\hat{s}(k)+u(k)-\hat{d}(k)
\end{aligned}
$$


Equation (10) represents the mean variation of inventory at each period $k, k \in\{1,2, \ldots . ., N-1\}$. Furthermore, $u(k)$ is deterministic, since it does not depend on the random variables $d(k)$ and $s(k)$. That is, $E\{u\}=u(k)$ with $V(u(k))=0 \quad \forall k$. Taking the difference between (1) and (10):

$$
\begin{gathered}
s(k+1)-\hat{s}(k+1)=s(k)-\hat{s}(k)-(d(k)-\hat{d}(k)) \\
\Rightarrow(s(k+1)-\hat{s}(k+1))^{2}=((s(k)-\hat{s}(k))-(d(k)-\hat{d}(k)))^{2} \\
\Rightarrow E\left((s(k+1)-\hat{s}(k+1))^{2}\right)=E\left(((s(k)-\hat{s}(k))-(d(k)-\hat{d}(k)))^{2}\right) \\
\Rightarrow E\left((s(k+1)-\hat{s}(k+1))^{2}\right)=E\left((s(k)-\hat{s}(k))^{2}+(d(k)-\hat{d}(k))^{2}-2 \cdot(s(k)-\hat{s}(k)) \cdot(d(k)-\hat{d}(k))\right) \\
\Rightarrow E\left((s(k+1)-\hat{s}(k+1))^{2}\right)=E\left((s(k)-\hat{s}(k))^{2}\right)+E\left((d(k)-\hat{d}(k))^{2}\right)-2 \cdot E((s(k)-\hat{s}(k)) \cdot(d(k)-\hat{d}(k)))
\end{gathered}
$$

Since $s(k)$ and $d(k)$ are independent random variables we can deduce that:

$$
E((s(k)-\hat{s}(k)) \cdot(d(k)-\hat{d}(k)))=E((s(k)-\hat{s}(k))) \cdot E((d(k)-\hat{d}(k)))
$$

Also, it is easy to see that:

$$
\begin{gathered}
E((s(k)-\hat{s}(k)))=E(s(k))-E(\hat{s}(k))=0 \\
E((d(k)-\hat{d}(k)))=E(d(k))-E(\hat{d}(k))=0
\end{gathered}
$$

Consequently,

$$
\begin{gathered}
E\left((s(k+1)-\hat{s}(k+1))^{2}\right)=E\left((s(k)-\hat{s}(k))^{2}\right)+E\left((d(k)-\hat{d}(k))^{2}\right) \\
V_{s}(k+1)=V_{s}(k)+V_{d}(k)=V_{s}(k)+\sigma_{d_{k}}^{2}
\end{gathered}
$$

If we assume that $V_{s}(k=0)=0$ and $\sigma_{d}(k)$ is constant and equal to $\sigma_{d}$ for all $k$ 's, we can deduce that: 


$$
\begin{gathered}
V_{s}(k)=k \cdot \sigma_{d_{k}}^{2} \\
\Rightarrow E\left((s(k)-\hat{s}(k))^{2}\right)=E\left(s(k)^{2}\right)-\hat{s}(k)^{2} \\
\Rightarrow E\left(s(k)^{2}\right)-\hat{s}(k)^{2}=V_{s}(k)=k \cdot \sigma_{d}^{2}
\end{gathered}
$$

Thus

$$
E\left(s(k)^{2}\right)=k \cdot\left(\sigma_{d}\right)^{2}+\hat{s}(k)^{2}
$$

Substituting (11) in the expected cost (8):

$$
\begin{gathered}
F(u)=C_{s} \times\left(\hat{s}(N)^{2}\right)+\sum_{k=0}^{N-1}\left[C_{s} \cdot \hat{s}(k)^{2} \times+C_{p r} \times u(k)^{2}\right]+C_{s} \times\left(\sigma_{d}\right)^{2} \times \sum_{k=0}^{N} k \\
\Rightarrow F(u)=C_{s} \times\left(\hat{s}(N)^{2}\right)+\sum_{k=0}^{N-1}\left[C_{s} \cdot \hat{s}(k)^{2} \times+C_{p r} \times u(k)^{2}\right]+C_{s} \times\left(\sigma_{d}\right)^{2} \times \frac{N(N+1)}{2}
\end{gathered}
$$

- $\quad$ The service level constraint (2):

Another step toward transforming the problem into a deterministic equivalent is to cast the service level constraint in a deterministic form by specifying certain minimum cumulative production quantities that depend on the service level requirements. It is necessary first to determine the change of the variance of inventory over the planning horizon.

\section{Lemma 2:}

$$
\operatorname{Pr} o b(s(k+1) \geq 0) \geq \alpha \Rightarrow\left(u(k) \geq U_{\alpha}(s(k), \alpha)\right) \quad k=0,1, \ldots ., N-1
$$

where

$U_{\alpha}()$ : Minimum cumulative production quantity

$$
U_{\alpha}(s(k), \alpha)=V_{d, k} \cdot \varphi_{d, k}^{-1}(\alpha)+\hat{d}(k)-s(k) \quad k=0,1, \ldots, N-1
$$

$V_{d, k}:$ Variance of demand $d$ at period $k$

$\varphi_{d, k}$ : Cumulative Gaussian distribution function with mean $\hat{d}_{k}$ and finite variance $\operatorname{Var}\left(d_{k}\right)=V_{d, k} \geq 0$

$\varphi_{d, k}^{-1}:$ Inverse distribution function

Proof of lemma1:

$$
s(k+1)=s(k)+u(k)-d(k)
$$




$$
\begin{gathered}
\Rightarrow \operatorname{Prob}(s(k+1) \geq 0) \geq \alpha \\
\Rightarrow \operatorname{Prob}(s(k)+u(k)-d(k) \geq 0) \geq \alpha \\
\Rightarrow \operatorname{Prob}(s(k)+u(k) \geq d(k)) \geq \alpha \\
\Rightarrow \operatorname{Prob}(s(k)+u(k)-\hat{d}(k) \geq d(k)-\hat{d}(k)) \geq \alpha \\
\Rightarrow \operatorname{Prob}\left(\frac{s(k)+u(k)-\hat{d}(k)}{V_{d, k}} \geq \frac{d(k)-\hat{d}(k)}{V_{d, k}}\right) \geq \alpha
\end{gathered}
$$

Note that $\left(\frac{d(k)-\hat{d}(k)}{V_{d, k}}\right)$ is a Gaussian random variable with an identical distribution as $d(k)$.

It is possible from (12) to determine a lower bound for the control variable, assuming that $\varphi$ is a probability distribution function and $f$ a probability density function. Hence,

$$
(12) \Rightarrow \varphi_{d, k}\left(\frac{s(k)+u(k)-\hat{d}(k)}{V_{d, k}}\right) \geq \alpha
$$

Since $\lim _{-\infty} \varphi_{d, k} \rightarrow 0$ and $\lim _{+\infty} \varphi_{d, k} \rightarrow 1$ we conclude that $\varphi_{d, k}$ is strictly increasing. We note that $\varphi_{d, k}$ is indefinitely differentiable, so we conclude that $\varphi_{d, k}$ is invertible.

$$
\begin{gathered}
\text { Thus }(13) \Rightarrow \frac{s(k)+u(k)-\hat{d}(k)}{V_{d, k}} \geq \varphi_{d, k}^{-1}(\alpha) \\
\Leftrightarrow s(k)+u(k)-\hat{d}(k) \geq V_{d, k} \cdot \varphi_{d, k}^{-1}(\alpha) \\
\Leftrightarrow u(k) \geq V_{d, k} \cdot \varphi_{d, k}^{-1}(\alpha)+\hat{d}(k)-s(k)
\end{gathered}
$$

Thus

$$
\operatorname{Prob}(s(k+1) \geq 0) \geq \alpha \Rightarrow\left(u(k) \geq V_{d, k} \cdot \varphi_{d, k}^{-1}(\alpha)+\hat{d}(k)-s(k)\right)
$$

This completes the proof.

Using Lemma 1 and Lemma 2, the equivalent deterministic model can now be formulated as follows: 


$$
\operatorname{Min}_{u} C_{s} \times\left(\hat{S}(N)^{2}\right)+\sum_{k=0}^{N-1}\left[C_{s} \cdot \hat{S}(k)^{2} \times+C_{p r} \times u(k)^{2}\right]+C_{s} \times\left(\sigma_{d}\right)^{2} \times \frac{N(N+1)}{2}
$$

Subject to:

$$
\begin{gathered}
\hat{s}(k+1)=\hat{s}(k)+u(k)-\hat{d}(k) \quad k=0,1, \ldots, N-1 \\
u(k) \geq V_{d, k} \cdot \varphi_{d, k}^{-1}(\alpha)+\hat{d}(k)-S(k) \quad k=0,1, \ldots, N-1 \\
0 \leq u(k) \leq U_{\max } \quad k=0,1, \ldots, N-1
\end{gathered}
$$

\section{III.2. Optimal maintenance plan considering the influence of the production plan on the deterioration of the manufacturing system}

For the maintenance policy, we seek to find the cost associated with a given schedule of future preventive maintenance and replacement activities. The joint optimization strategy considers these costs based on optimal production rates previously found by the production policy in order to optimize the maintenance strategy characterized by the optimal time interval between successive preventive maintenance or replacement activities, $k^{*} \Delta t$.

The analytic expression of the average cost per unit time of maintenance actions is defined by:

$$
C(k)=\frac{C_{p}+C_{c} \times A_{k}}{k \cdot \Delta t}
$$

Where $A_{k}$ corresponds to the expected number of failure, i.e. the average number of failures that can occur during the horizon $H$, considering the production rate variation for each production period $\Delta t$. We recall that the manufacturing system considered in this study is composed of a machine $M$ which produces a single product at the rate $u(k)$ during each $\Delta t$ period with the reliability function $R_{k}(t, u(k))(k=0,1 \ldots N-1 ; N . \Delta t=H)$.

Since $u(k)$ varies in each production period $\Delta t$, it is complex to formulate directly the analytical expression of $A_{k}$, which is why we do so by employing the operational age method. Using the maximal production rate and the failure rate, i.e. the nominal failure rate, we determine the expected failure number as follows:

$$
A_{k}=\sum_{i=1}^{k} \int_{\Gamma_{i}}^{\Gamma_{i}+\Delta t} \lambda_{i}(t, u(i)) d t
$$

where

$$
\lambda_{i}(t, u(i))=\frac{u(i)}{U_{\max }} \cdot \lambda_{0}(t)
$$


Remark:

$\lambda_{0}(t)$ : the nominal failure rate

We assume that the nominal failure rate is the failure rate where the production level is maximal.

$\Gamma_{i}$ : Time at which the reliability at the end of period $i-l$ is identical to that at the beginning of the next period $i$ We now determine an analytical expression for $\boldsymbol{\Gamma}_{i}$.

\section{Lemma 3:}

$$
\Gamma_{j}=R_{j}^{-1}\left(R_{j-1}\left(\Gamma_{j-1}+\Delta t\right)\right) \quad \text { For } \quad j \geq 3
$$

$$
\left\{\begin{array}{l}
\Gamma_{2}=R_{2}^{-1}\left(R_{1}(\Delta t)\right) \\
\Gamma_{1}=0
\end{array}\right.
$$

$R_{\max }^{-1}$ : inverse of the reliability with the nominal (maximum) production.

$R_{i+1}$ : reliability at the production rate $u(i+1)$.

$\Gamma_{i+1}$ : time at which the reliability at the end of period $i$ is identical to that at the beginning of period $i+1$.

$\Gamma_{j}$ : time at which the reliability at the end of period $j-1$ is identical to that at the beginning of the next period $j$.

Proof:

The operational age model considers that the reliability associated with the beginning of the period $i+1$ is equal to the reliability at the end of the previous period $i$.

$$
R_{u_{i}}\left(t_{u_{i}}\right)=R_{u_{i+1}}\left(t_{u_{i}}\right)
$$

With

$t_{u_{i}}$ : the system age at the end of the period where the rate of production equal to $u(i)$

$R_{u i}$ : reliability at the production rate $u(i)$.

$R_{u i+1}$ : reliability at the production rate $u(i+1)$.

To verify that equation (knowing that each period characterized by a production rate). If a period characterized by a production rate $u(i)$ for a period $i . \Delta t$, is equivalent to the period characterized by a production rate $u(i+1)$, but during a different duration $d_{u i+1}$. This new duration characterizes the operational age system and the previous relation becomes:

$$
R_{u_{i}}\left(t_{u_{i}}\right)=R_{u_{i+1}}\left(\Gamma_{u_{i+1}}\right)
$$

Where

$\Gamma_{u_{j+1}}=\sum_{i=1}^{j} d_{u_{j}}$ is the operational age at the beginning of the next period $i+1$ characterized by the production rate $u(i+1)$. 
As production rates may vary between periods, the expected number of failure number is given by:

$$
A_{k}=\sum_{i=1}^{k} \int_{\Gamma_{i}}^{\Gamma_{i}+\Delta t} \lambda_{i}(t, u(i)) d t
$$

From equation (7):

$$
\begin{gathered}
\lambda_{i}(t, u(i))=\frac{u(i)}{U_{\max }} \cdot \lambda_{0}(t) \\
A_{k}=\sum_{i=1}^{k} \int_{\Gamma_{i}}^{\Gamma_{i}+\Delta t} \frac{u(i)}{U_{\max }} \cdot \lambda_{0}(t) d t
\end{gathered}
$$

where:

$\Gamma_{i}$ : time at which the reliability at the end of period $i-1$ is identical to that at the beginning of period $i$. $R_{i}\left(\Gamma_{i}\right)$ : reliability function associated with period $i$.

$R_{i}^{-1}\left(R_{i}\left(\Gamma_{i}\right)\right)$ : inverse of $R_{i}\left(\Gamma_{i}\right)$.

Fig 3. Policy example

Assuming continuity, we must have,

$$
R_{i}\left(\Gamma_{i}\right)=R_{i-1}\left(\Gamma_{i-1}+\Delta t\right) \quad i \geq 3
$$

and $R_{2}\left(\Gamma_{2}\right)=\left(R_{1}(\Delta t)\right)$, where $R_{i}$ denotes the reliability at the production rate $u(i) \leq U_{\max }$.

Figure 3 illustrates variable $\Gamma_{i}$

Fig 4. Schematic representation of $\boldsymbol{\Gamma}_{\boldsymbol{i}}$

Note that in Figure 3, we assume that $u_{i-1} \leq u_{i+1} \leq u_{i}$.

Since

$$
\begin{gathered}
g(u(i-1)) \leq g(u(i+1)) \leq g(u(i)) \\
\text { and } \quad \lambda_{i-1}(t, u(i-1)) \leq \lambda_{i+1}(t, u(i+1)) \leq \lambda_{i}(t, u(i))
\end{gathered}
$$


1

2

3

4

5

6

7

8

9

10

11

$\Gamma_{i}$ can be determined from equation (15),

$$
\Gamma_{i}=R_{i}^{-1}\left(R_{i-1}\left(\Gamma_{i-1}+\Delta t\right)\right) .
$$

This completes the proof.

Using Lemma 2 the maintenance cost can now be written as follows:

$$
C(k)=\frac{C_{p}+C_{c} \times \sum_{i=1}^{k} \int_{\Gamma_{i}}^{\Gamma_{i}+\Delta t} \lambda_{i}(t, u(i)) d t}{k \cdot \Delta t}
$$

The minimum maintenance cost is obtained by solving the following equation which yields $k$.

$$
\frac{\partial C(k)}{\partial k}=0
$$

Lemma 4 proves the existence of a local minimum.

\section{Lemma 4:}

$$
\exists k^{*} \text { if } \theta_{k-1} \leq \frac{C_{p}}{C_{c}} \leq \theta_{k}
$$

With

$$
\theta_{k}=k \cdot A_{k+1}-(k+1) \cdot A_{k}
$$

Proof:

We recall that:

with

$$
\begin{gathered}
C(k)=\frac{C_{p}+C_{c} \times A_{k}}{k \cdot \Delta t} \\
A_{k}=\sum_{i=1}^{k} \int_{\Gamma_{i}}^{\Gamma_{i}+\Delta t} \frac{u(i)}{u_{\max }} \cdot \lambda_{0}(t) d t
\end{gathered}
$$

Since we have: 


$$
\begin{aligned}
& \text { - } C(k+1)-C(k)=\frac{C_{p}+C_{c} \times A_{k+1}}{(k+1) \cdot \Delta t}-\frac{C_{p}+C_{c} \times A_{k}}{k \cdot \Delta t} \\
& =\frac{C_{c} \times\left(k \times A_{k+1}-(k+1) \times A_{k}\right)-C_{p}}{k(k+1) \cdot \Delta t} \\
& C((k+1))-C(k) \geq 0 \\
& \frac{C_{c} \times\left(k \times A_{k+1}-(k+1) \times A_{k}\right)-C_{p}}{k(k+1) \cdot \Delta t} \geq 0 \\
& C_{c} \times\left(k \times A_{k+1}-(k+1) \times A_{k}\right)-C_{p} \geq 0 \\
& \left(k \times A_{k+1}-(k+1) \times A_{k}\right) \geq \frac{C_{p}}{C_{c}} \\
& \text { - } C(k)-C(k-1)=\frac{C_{p}+C_{c} \times A_{k}}{k \cdot \Delta t}-\frac{C_{p}+C_{c} \times A_{k-1}}{(k-1) \cdot \Delta t} \\
& =\frac{C_{c} \times\left((k-1) \times A_{k}-k \times A_{k-1}\right)-C_{p}}{k(k+1) \cdot \Delta t} \\
& C(k)-C((k-1)) \leq 0 \\
& \frac{C_{c} \times\left((k-1) \times A_{k}-k \times A_{k-1}\right)-C_{p}}{k(k+1) \cdot \Delta t} \leq 0 \\
& C_{c} \times\left((k-1) \times A_{k}-k \times A_{k-1}\right)-C_{p} \leq 0 \\
& \left((k-1) \times A_{k}-k \times A_{k-1}\right) \leq \frac{C_{p}}{C_{c}}
\end{aligned}
$$

When the failure time has a Weibull distribution, i.e., $A_{k}(t)=a \cdot t^{\gamma} \quad(\gamma>1)$ and $a>0$

$$
\begin{gathered}
(17) \Rightarrow k \times A_{k+1}-(k+1) \times A_{k} \geq \frac{C_{p}}{C_{c}} \\
\Rightarrow k \times a \times(k+1)^{\gamma}-(k+1) \times a \times k^{\gamma} \geq \frac{C_{p}}{C_{c}} \\
\Rightarrow k \times(k+1)^{\gamma}-(k+1) \times k^{\gamma} \geq \frac{1}{a} \times \frac{C_{p}}{C_{c}}
\end{gathered}
$$

Since it is easily proved that $k \times(k+1)^{\gamma}+(k+1) \times k^{\gamma}$ is strictly increasing in $k \rightarrow \infty$ and $\gamma-1>0$ 


$$
\begin{aligned}
(18) & \Rightarrow(k-1) \times A_{k}-k \times A_{k-1} \leq \frac{C_{p}}{C_{c}} \\
& \Rightarrow(k-1) \times a \times k^{\gamma}-k \times a \times(k-1)^{\gamma} \leq \frac{C_{p}}{C_{c}} \\
& \Rightarrow(k-1) \times k^{\gamma}-k \times(k-1)^{\gamma} \leq \frac{1}{a} \times \frac{C_{p}}{C_{c}}
\end{aligned}
$$

The function $(k-1) \times k^{\gamma}-k \times(k-1)^{\gamma}$ is strictly decreasing in $k \rightarrow 1$ and $\gamma-1>0$.

Thus:

$$
k^{*}=\left\{\begin{array}{l}
C(k+1)-C(k) \geq 0 \\
C(k)-C(k-1) \leq 0 \\
C(k+1)-C(k) \text { is increasing in in } k \rightarrow+\infty \\
C(k)-C(k-1) \text { is decreasing in } k \rightarrow 1
\end{array}\right.
$$

$\Rightarrow$ Therefore, there exists as the production period where preventive maintenance should be performed Since it is complex to solve equation (16) analytically, we next develop a numerical procedure for doing so which we illustrate via a numerical example.

\section{Optimal production and maintenance plans: A numerical example}

In this section, the development of joint production-maintenance plans for a hypothetical company is introduced as an example. It is assumed that this company manufactures one product type whose demand fluctuates periodically. It is assumed that a production plan is generated for a planning horizon $H=18$ months, and that the failure time of machine $M$ is characterized by a Weibull distribution with increasing failure rate, implying the existence of an optimal maintenance schedule.

The main data of the problem are:

(i) the monthly mean demands $\hat{d}_{k}$ are given by the sequence :

Table 1. The mean demands

(ii) $\quad C_{p r}=3 m u, C_{s}=2 m u$

(iii) $\quad U_{\min }=2 \mathrm{ut}$ and $U_{\max }=10 \mathrm{ut}$ 
(iv) $\quad S(0)=10 u t$

(v) $d_{k}$, which is extracted from a historical sales report, is assumed Gaussian with $\sigma_{d}=1.42$

(vi) The degree of customer satisfaction, associated with the service level constraint (2), is equal to $90 \%$ $(\alpha=0.9)$.

In order to solve $(P 1)$ a numerical procedure consisting of dynamic programming is developed. Due to the additive structure of the functional production/inventory cost, the principle of optimality can be applied and, as a result, a sequence of sub problems can be defined and solved interactively during the horizon $H$. The problem $(P 1)$ becomes one of finding a sequence of control $\left\{u_{k} \in U_{\alpha}=\left[\max \left(U_{\min }, u_{\alpha}(S(k), \alpha), U_{\max }\right], k=0,1, \ldots, N-1\right\}\right.$ where $U_{\alpha}$ is a sub-space that according to the observed state and the probability measure $\alpha$ at each period $k$.

The optimal production plan and the optimal maintenance period are exhibited respectively in Table 2 and Figure 5 .

Table 2. Optimal production plan

For the maintenance policy, the scale and shape parameters of the Weibull distribution are respectively $\beta=16.79$ and $\delta=3$, while $C_{c}=3000 \mathrm{mu}, C_{p}=500 \mathrm{mu}$, and $\Delta t=1$.

We invoked Lemma 3 using the numerical data, which yielded the following:

$$
\Gamma_{j}=\left(\frac{u(j-1)}{u(j)}\right)^{\frac{1}{\delta}} \cdot\left(\Gamma_{j-1}+\Delta t\right) \quad j \geq 3
$$

and

$$
\left\{\begin{array}{l}
\Gamma_{2}=\left(\frac{u(1)}{u(2)}\right)^{\frac{1}{\delta}} \cdot \Delta t \\
\Gamma_{1}=0
\end{array}\right.
$$

Fig 5. Curve of the average total maintenance cost as a function of $k$ assuming optimal production rates

Figure 5 presents the curve of the average total maintenance cost per time unit, $C(k)$, as a function of $k$. We observe that the optimal preventive maintenance period is $k^{*} \Delta t=8 \Delta t$ with a corresponding optimal cost of $C^{*}=111.114 \mathrm{mu}$.

Fig 6. Curve of the average total maintenance cost as a function of $k$ assuming the maximum production rate

Previous research assumed nominal (maximal) production rates in devising maintenance policies, corresponding to the result exhibited in Figure 6 for the numerical example at hand. By contrast, Figure 5 reveals the cost reduction engendered by using optimal instead of nominal production rates, in the order of $6 \%$ in this case. 


\section{References}

Akella, R. \& Kumar, P.R. (1986) .Optimal control of production rate in a failure-prone manufacturing system,IEEE Transactions on Automatic Control, AC-31. 116-126.

Brown, M., \& Proschan. F. (1983). Imperfect repair. Journal of Applied Probability ,20 (4). 851-859.

Boukas, E.k,\& Haurie, A. (1990). Manufacturing flow control and preventive maintenance:a stochastic control approach,IEEE Transaction on Automatic control,35 1024-1031.

Boukas, E.k, \& Yang, H.,(1996). Optimal control of manufacturing flow and preventive maintenance, IEEE Transactions on Automatic Control ,41 881-885.

Barták, R., Salido, M. A., \& Rossi, F. (2009). Constraint satisfaction techniques in planning and scheduling. Journal of Intelligent Manufacturing. doi:10.1007/s10845-008-0203-4.

Cheung, K. L \& Hausmann, W. H. (1997) . Joint optimization of preventive maintenance and safety stock in an unreliable production environment. Naval Research Logistics, 44: 257-272.

Cox D.R. (1972). Regression Models and Life-Tables. Journal of the Royal Statistical Society. 34 (2). 187220.

Dellagi, S., N. Rezg, X. Xie,(2007) . Preventive Maintenance of Manufacturing Systems Under Environmental Constraints, International Journal of Production Research, vol. 45, Issue 5, p1233-1254.

Kijima, M., Morimura, H. \& Suzuki Y. (1988). Periodical replacement problem without assuming minimal repair. European Journal of Operational Research, 37. 194-203.

Faulkner,L.L.(2005).Maintenance,Replacement,andReliabilityTheoryand pplications.Chapter 2 Component Replacement Decisions, Taylor \& Francis Group,USA.

Martorell S., Ana Sanchez S., Serradell V. (1999) Age dependent reliability model considering effects of maintenance and working conditions. Reliability Engineering \& System Safety, Volume 64, Issue 1: 19-31.

O.S.Silva, F. \& Cezarino .W. (2004).An Optimal Production Policy applied to a Flow-shop Manufacturing System. Brazilian Journal of Operations \& Production Management, Brazil.

Rezg, R.,Dellagi, S \& Chelbi, A. (2008) Optimal Strategy of Inventory Control and Preventive Maintenance. International Journal of Production Research ,Vol. 46 No. 19: 5349-5365.

Rezg, N.,Xie,X.,\& Mati,Y (2004) Joint optimization of preventive maintenance and inventory control in a production line using simulation. International Journal of Production Research 44:2029-2046.

Schutz, J. , Rezg, N.\&J.-B. Léger.(2009) Periodic and sequential preventive maintenance policies over a finite planning horizon with a dynamic failure law. Journal of Intelligent Manufacturing, doi:10.1007/s10845009-0313-7.

Van der Duyn Schouen, F.A., and Vanneste, S.G.(1995) .Maintenance optimization of a production system with buffer capacity,European Journal of Operational Research, 82,323-338.

(Özekici) Özekici, S. (1995) Optimal maintenance policies in random environments. European Journal of Operational Research, Volume 82, Issue 2: 283-294. 


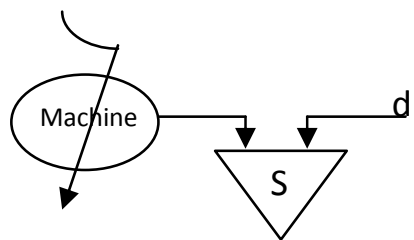

Fig 1. Problem description

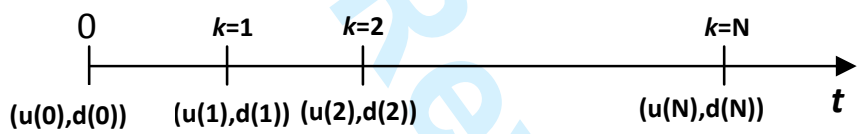

Fig 2. Discrete time 


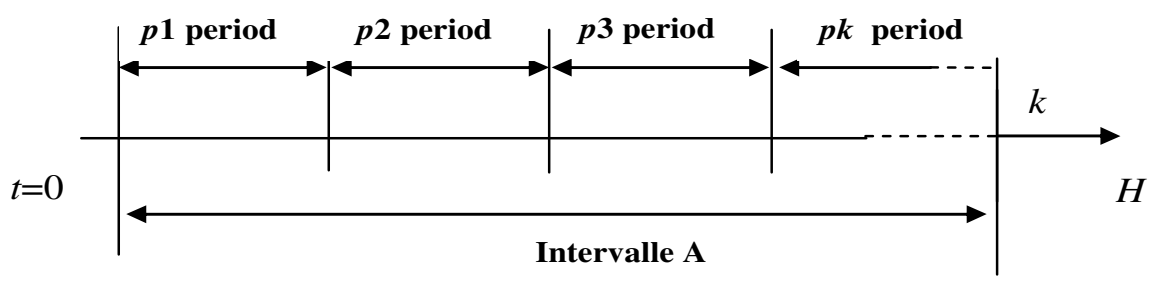

Fig 3. Policy example

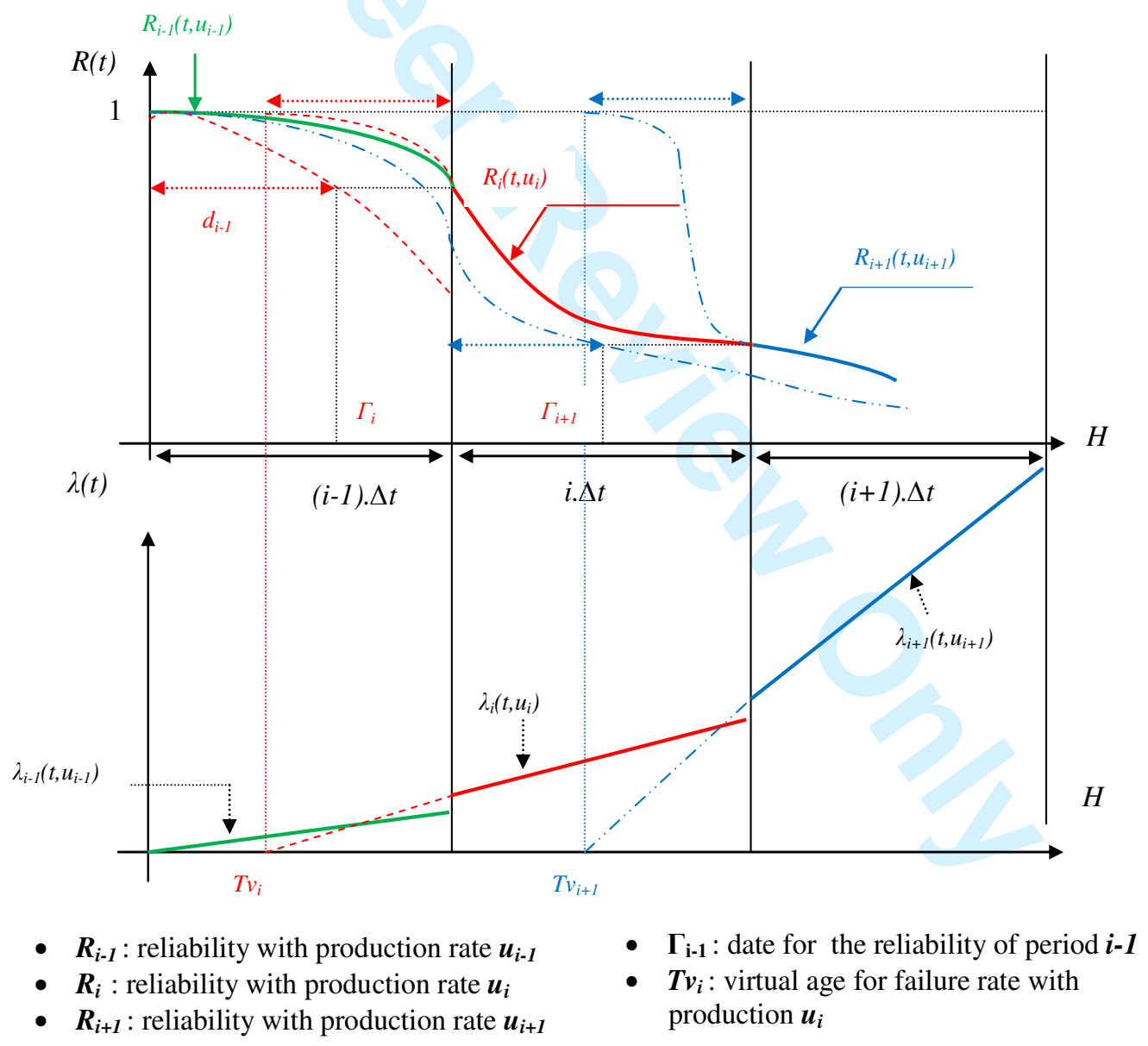

Fig 4. Schematic representation of $\boldsymbol{\Gamma}_{\boldsymbol{i}}$ 


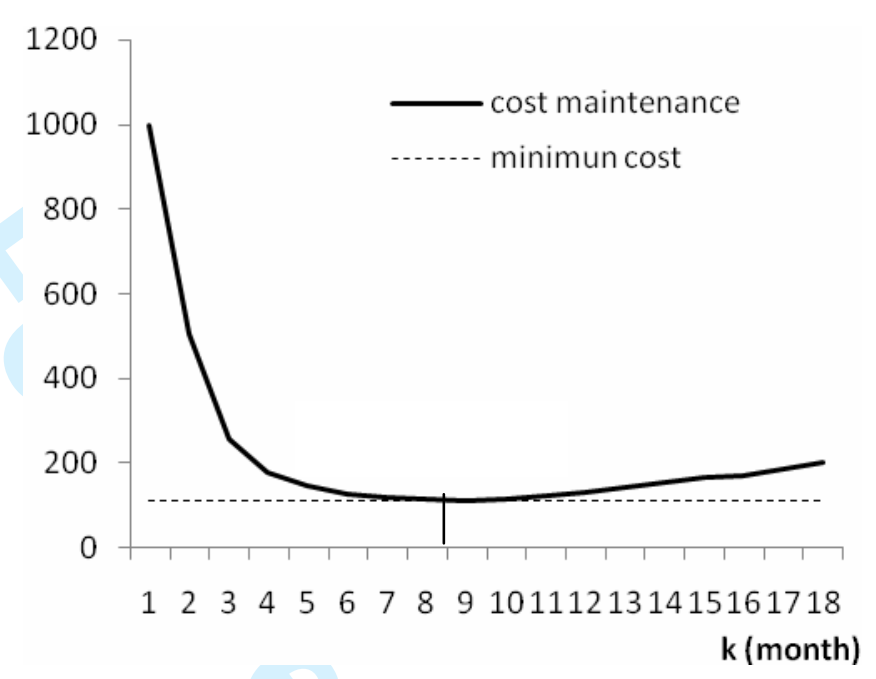

Fig 5. Curve of the average total maintenance cost as a function of $k$ assuming optimal production rates

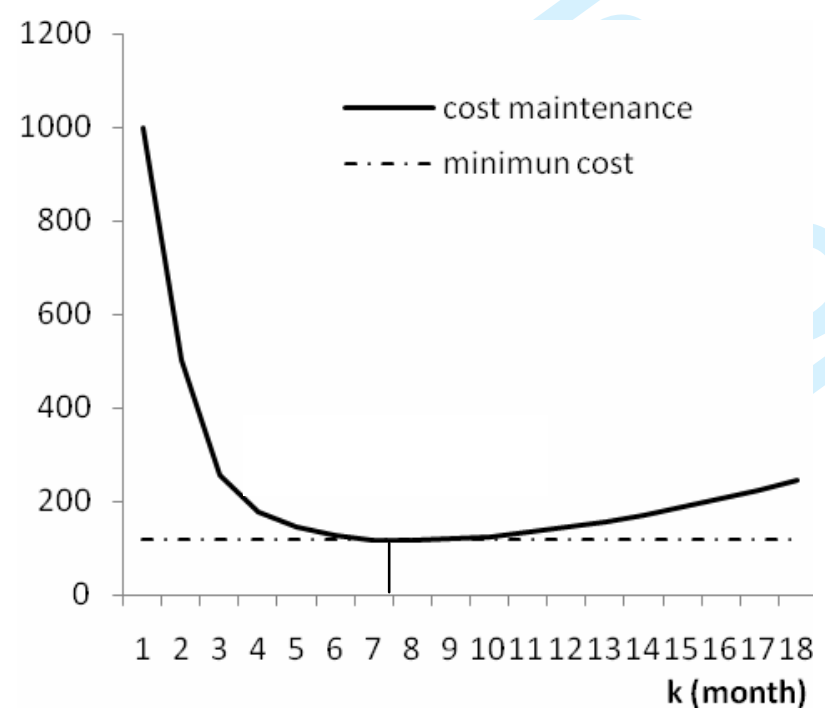

Fig 6. Curve of the average total maintenance cost as a function of $k$ assuming the maximum production rate 


\begin{tabular}{|c|c|c|c|c|c|c|c|c|}
\hline$d_{1}$ & $d_{2}$ & $d_{3}$ & $d_{4}$ & $d_{5}$ & $d_{6}$ & $d_{7}$ & $d_{8}$ & $d_{9}$ \\
\hline $\mathbf{8}$ & $\mathbf{8}$ & $\mathbf{9}$ & $\mathbf{8}$ & $\mathbf{8}$ & $\mathbf{8}$ & $\mathbf{7}$ & $\mathbf{6}$ & $\mathbf{4}$ \\
\hline$d_{10}$ & $d_{11}$ & $d_{12}$ & $d_{13}$ & $d_{14}$ & $d_{15}$ & $d_{16}$ & $d_{17}$ & $d_{18}$ \\
\hline $\mathbf{5}$ & $\mathbf{7}$ & $\mathbf{8}$ & $\mathbf{1 0}$ & $\mathbf{8}$ & $\mathbf{9}$ & $\mathbf{5}$ & $\mathbf{6}$ & $\mathbf{6}$ \\
\hline
\end{tabular}

Table 1: the mean demands

\begin{tabular}{|c|c|c|c|c|c|c|c|c|c|}
\hline$k$ & 1 & 2 & 3 & 4 & 5 & 6 & 7 & 8 & 9 \\
\hline $\boldsymbol{d}$ & 8 & 8 & 9 & 8 & 8 & 8 & 7 & 6 & 4 \\
\hline$u^{*}(k)$ & $\mathbf{1 0}$ & $\mathbf{1 0}$ & $\mathbf{1 0}$ & $\mathbf{9}$ & $\mathbf{8}$ & $\mathbf{8}$ & $\mathbf{5}$ & $\mathbf{4}$ & $\mathbf{2}$ \\
\hline $\boldsymbol{k}$ & 10 & 11 & 12 & 13 & 14 & 15 & 16 & 17 & 18 \\
\hline $\boldsymbol{d}$ & 5 & 7 & 8 & 10 & 8 & 9 & 5 & 6 & 6 \\
\hline $\boldsymbol{u}^{*}(\boldsymbol{k})$ & $\mathbf{5}$ & $\mathbf{1 0}$ & $\mathbf{1 0}$ & $\mathbf{1 0}$ & $\mathbf{9}$ & $\mathbf{1 0}$ & $\mathbf{2}$ & $\mathbf{4}$ & $\mathbf{6}$ \\
\hline
\end{tabular}

Table 2. Optimal production plan 